\title{
Museo Urbano: Planteamiento conceptual que integra la conexión, exposición e interacción con material cultural en espacios públicos, en la Avenida Central, San José.
}

\author{
Sofía Rodríguez Cerdas \\ Escuela de Arquitectura, Universidad de Costa Rica. \\ San Pedro de Montes de Oca, San José, Costa Rica \\ E-mail: sofiarc015@gmail.com,
}

\begin{abstract}
Resumen. Museo Urbano, es un planteamiento conceptual desde el cual se logra entender el espacio público como un medio dispuesto a conectar, comunicar y gestar las diversas interacciones sociales y múltiples actividades desarrolladas en este, para la puesta en valor de la identidad urbana.

Este constructo se ha desarrollado en el marco de la investigación del trabajo final de graduación para optar por el título de Licenciatura en Arquitectura. El trabajo se desarrolló mediante la indagación de referentes teóricos. En primera instancia, se estudió el constructo espacio público como hábitat común, que involucra la confrontación y convivencia humana. Posteriormente se analizó el concepto de museo, con el fin de ser deconstruido, como campo de comunicación entre la gente y su contenido; enfocándolo en el otorgar experiencias como medio social e interactivo.

La investigación toma como laboratorio de análisis el eje peatonal de la Avenida Central en San José, Costa Rica, diagnosticando su situación en términos de las dinámicas que alli se gestan. A partir de ello, se desarrolla un plan estratégico que identifica acciones y transformaciones para la visualización del planteamiento en ese eje central de la ciudad de San José.

Museo Urbano evidencia escenas y componentes urbanos como relatores de los valores cotidianos de la ciudad y que conducen a entender la cultura como componente intrínseco del espacio público. Es a través de la valoración del espacio público como Museo que se justifica también la necesidad de gestión del mismo, para fortalecer los valores físicos y simbólicos de la ciudad.
\end{abstract}

Palabras clave: Espacio público, museo, Avenida Central, cultura, vida urbana, interacción, conexión, comunicación

\section{Previo a Museo Urbano: Óptica de lectura del espacio público}

\section{* Equivalencia: $M U R=$ Museo Urbano}

La toma de decisiones al proyectar y desarrollar espacios públicos en gran cantidad de urbes, no ha considerado la facultad del mismo como plataforma de expresión en la ciudad. De esta manera se han expuesto "medidas de control" que persuaden la ilegitimización de grupos de personas y actividades; negando la existencia de múltiples realidades como sociedad.
"Lo que bien podría reconocerse como el idealismo del espacio público aparece hoy al servicio de la reapropiación capitalista de la ciudad, una dinámica de la que los elementos fundamentales y recurrentes son la conversión de grandes sectores del espacio urbano en parques temáticos, la gentrificación de centros históricos de los que la historia ha sido definitivamente expulsada, la reconversión de barrios en industrias enteras, la dispersión de una miseria creciente que no se consigue ocultar, el control sobre un espacio público cada vez menos público, etc." (Delgado, 
Manuel. 2011:10)

El problema que enfrentó la investigación, está constituido por la falta de estrategias conceptuales, decisiones y acciones que potencien el espacio público como medio de exposición, conexión e interacción para todas $\mathrm{y}$ todos, desde la perspectiva de confrontación y adaptación social.

Razón por la cual, se desarrolla una óptica de lectura y entendimiento de la ciudad, mediante la comunicación y apropiación de la misma, para facilitar la activación urbana. De esta manera, se pretende liberar "material cultural" de manera gratuita y democrática a la gente, con el fin de impactar desde la experiencia de interacción y contacto social.

El planteamiento surge a partir de un cuerpo conceptual que identifica fundamentos, ejes de acción y bases para analizar el espacio público en torno a su concepción. Tiene potencial de ser estudiado en otros centros urbanos, lo que da cabida a la reinterpretación del mismo de acuerdo a: emplazamiento, infraestructura, modo de vida, caracterización identitaria, entre otras.

La estructura conceptual de Museo Urbano, puede ser de beneficio para organizaciones, instituciones y gobiernos locales, que busquen establecer alternativas de reactivación del espacio público.

1.1 ¿Hacia dónde se dirige Museo Urbano?

La existencia de propuestas por parte de organizaciones e instituciones (en distintas ciudades y países) que pretenden generar activación urbana, señala la disposición de gobiernos locales por implementar estrategias de impacto cultural. Sin embargo, no exponen alcances que traten el espacio público de manera protagónica; mediante la diversificación de actividades para la convivencia humana.

¿Cómo es posible entender y visualizar el espacio público a través del priorizar la conexión entre espacios de encuentro, exposición de material, actividades y situaciones urbanas, aunado a la interacción social; desde la óptica de visibilización cultural?

Por lo tanto, MUR constituye una serie de herramientas y conceptos interpretables, para observar la burbuja pública como zona de coexistencia y visibilización en las ciudades.

1.3 Estrategia metodológica de investigación

El presente documento se expone mediante la articulación de cuatro bloques principales como estrategia de estudio para abordar el problema. Lo anterior resultó de un proceso evolutivo, que orientó la investigación hacia la definición del planteamiento conceptual.

1.3.a Bloque origen: Constituye la etapa introductoria de recopilación de información en cuanto a referentes teóricos sobre espacio público y museo, además el análisis de antecedentes en términos de cultura, ciudad y gente.

1.3.b Bloque Construcción del cuerpo teórico: En este apartado se definió el planteamiento conceptual Museo Urbano, a través de la interpretación de insumos teóricos, que permitieron desarrollar una óptica de observación y análisis del espacio público.

1.3.c Bloque Avenida Central: Laboratorio de análisis: Se enfocó en la observación y comprensión de eje peatonal principal de la ciudad de San José, como elemento urbano dotado de gran cantidad de funciones, situaciones y condiciones físicas, articuladas mediante su valor identitario.

1.3.c Bloque Aportes desde el planteamiento: La última unidad de investigación, desarrolló como primer insumo un plan estratégico que propuso actividades e intervenciones para Museo Urbano, en el caso de la Avenida Central. Finalmente expuso una serie de conclusiones como aportes del planteamiento en relación al espacio público y sitio de estudio. Lo que representa inferencias que pueden ser utilizadas posteriormente, para llevar a cabo el proyecto en la Avenida Central o en otros contextos urbanos.

\section{Museo Urbano: Construcción teórico- conceptual}

El desarrollo del cuerpo teórico se ejecutó mediante la interrelación de conceptos, por un lado de espacio público como esfera social, cultural y física y por el otro, de museo, como elemento integrador anuente a generar experiencias mediante la interacción con su contenido.

En primera instancia, se analizaron planteamientos teóricos sobre espacio público 
para ser entendido como conector y construir la redefinición del término "paseo", que vincula espacio, gente y acontecimientos (desde el enfoque cultural).

A partir de lo anterior, se generó una discusión tomando en cuenta referencias teóricas de índole socio-cultural, para poner en manifiesto parte de la situación actual de la Avenida Central en San José, como laboratorio de investigación. De esta manera, se construyó el concepto "paseo cultural", el cual fue posicionado como observación crítica al actual Paseo de los Museos en San José.

El museo fue analizado desde su desarrollo como ente público con enfoque pedagógico. Para efectos de la investigación, se realizó la deconstrucción de este concepto o planteamiento, con el fin de dotarlo de nuevos significados que involucren mayor interacción humana $\mathrm{y}$ eventualmente, le permita ser entendido como espectro, con capacidad de análisis en múltiples contextos sin depender del marco institucional.

Finalmente el subcapítulo de museo urbano, surgió mediante el vínculo de los anteriores, para relacionar dos elementos de distinta naturaleza, museo y espacio público, como estrategia de interacción cultural-física y simbólica en la ciudad. (Imagen 1)

\section{Espacio público: Esfera de confrontación y convivencia}

A partir de los subcapítulos desarrollados en la investigación, se entiende el espacio público como articulador de distintas dimensiones humanas: -física, socio-cultural, económica, política, jurídica, otras-; mediante el análisis de componentes perceptuales y la descripción de elementos y procesos del entorno.

Se observaron distintas cualidades y componentes físicos-urbanos que definen la burbuja pública, orientados en cómo estos intervienen en la lectura, percepción e interacción entre la gente; ocasionando distintos tipos de permanencias y actividades.

Sin embargo, a la dimensión sociocultural se le atribuyó mayor carácter, por el impacto que tiene en el reconocimiento del espacio público. El contacto entre personas, su posible interacción y expresividad urbana, son situaciones fácilmente identificables por su

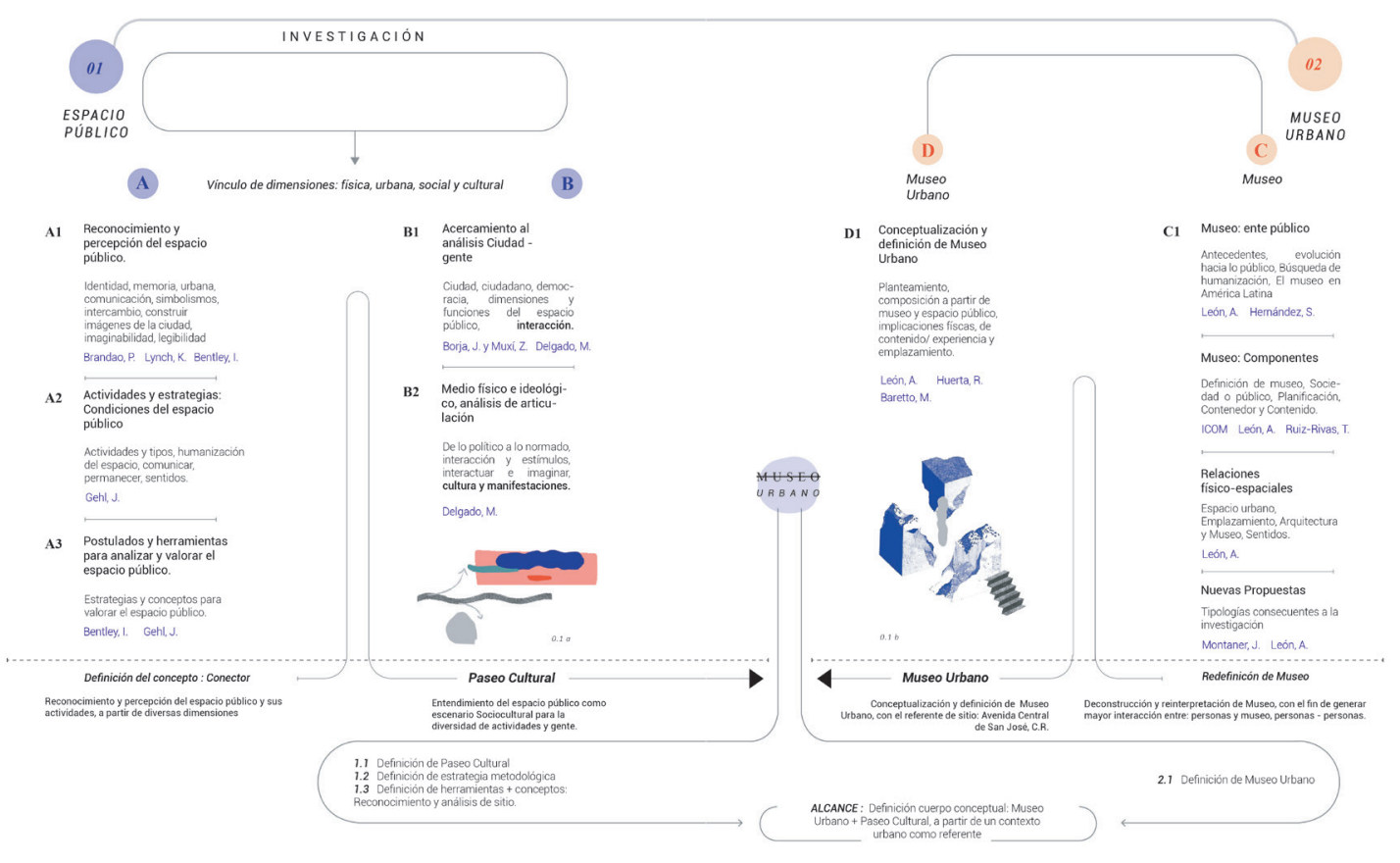

Fig. 1. (Imagen 1) Construcción teórico-conceptual Museo Urbano (Rodríguez, Sofía. 2018) 
valor identitario en la comunicación, que dan lugar a la coexistencia.

\section{Paseo Cultural: Estrategia de conexión en el centro de la ciudad de San José}

La investigación desarrolló una serie de conceptos asociados al constructo Museo Urbano, de esta manera, cabe destacar la concepción de Paseo Cultural, como contrapropuesta hacia iniciativas de alianza entre museos y gobierno local, dadas en el centro de la ciudad de San José (tomada como estudio de caso).

La representación como contrapropuesta, surge luego de observar y analizar cómo se direcciona el término "turismo cultural", a partir de una serie de representaciones culturales/académicas/elitistas, que no involucran el contacto con las y los habitantes de la ciudad, sino con grupos de población con cierto alcance educativo y económico.

Paseo Cultural: Planteamiento con repercusiones espaciales a nivel urbano, que pretende relacionar personas, procesos culturales formales e informales (artísticos, escénicos, musicales, de lectura, entre otros) y componentes físico-infraestructurales pertenecientes a un sitio, mediante el vínculo entre espacio público, museos y sitios que actualmente funcionan como plataforma de expresión cultural (señalados más adelante); con el fin de permitir comunicación e interacción, entre personas y contenido.

La conexión cultural pretende establecer estímulos espaciales que vivifiquen la experiencia urbana, permitan reconocer y entrelazar diversos puntos de valor histórico, patrimonial y cultural a nivel de sitio.

\section{Museo: De institución a espectro}

El museo tiene potencial como elemento intermediario social, vitalizador, que facilita el contacto y entendimiento del material que expone, mediante el "cara a cara" entre personas y objeto. A partir de lo expuesto previamente es posible narrar el museo como campo de encuentro, como lo describe León, Aurora. (2010), debería ser acentuado y menos rígido.

De esta manera su visión estaría concentrada en generar experiencias de interacción que entran y salen del museo, otorgando acción protagónica a las personas, como interactuantes, de los cuales el museo aprende.

Lo anterior representa una síntesis del esfuerzo por deconstruir qué es museo, cómo se piensa, muta y revalora a lo largo del tiempo. Razón por la cual, a nivel de investigación, se llevó a cabo la revisión de documentos que narran cómo ha sido la evolución del mismo en diferentes países, haciendo énfasis en la región Latinoamericana. Además del estudio de conceptos asociados a este, que hacen referencia a contenido, contenedor, material, público, nuevas propuestas y desarrollos (como estudios de caso), entre otros.

\section{Repensar el museo}

El museo entre sus desafíos, debe orientarse a la búsqueda de apertura estructural como entidad normada, para representar un medio humano de comunicación inclusivo. Sus valores técnicos tienen capacidad de transmutar (dependiendo de ideología, objetivos y políticas) hacia mecanismos con mayor espontaneidad y posible interacción.

Es imprescindible observar la dinámica cultural de la región de estudio, en este caso Avenida Central y zonas aledañas, para que sean expresadas en el museo a nivel de metodología y contenido posteriormente. Además es conveniente abrir el panorama y buscar vínculos con otros entes, para enriquecer la experiencia del futuro planteamiento sobre Museo Urbano, lo que requiere involucrar el espacio público desde sus actividades.

Además se elaboró un esquema de conclusiones que comunica los alcances del museo, para fines de la presente investigación. Este se constituye una red de análisis entre el vínculo de términos expuestos anteriormente (sociedad, emplazamiento, alcances culturales y diversas manifestaciones); con el fin de obtener deducciones articuladas en torno a diversos temas de impacto con respecto al funcionamiento del museo. 


\section{Planteamiento conceptual Museo Urbano}

Museo Urbano se proyecta como propuesta a partir de la deconstrucción o resignificación del museo y su planteamiento e impacto en el espacio público. A partir de premisa la ciudad es para todas y todos, la propuesta contempla la participación de sectores de la población no tomados en cuenta usualmente y la presencia de distintas capacidades cognitivas y físicas.

Además reconoce al "público" del museo como interactuantes, personas con acción protagónica en los espacios de exposición, comunicación / zonas de encuentro o tránsito, en sus distintos caracteres.

\section{Composición a partir de dos ejes}

Los capítulos expuestos en la construcción conceptual de investigación, permitieron desarrollar el planteamiento museo urbano, mediante el vínculo y análisis del museo a partir de su evolución como institución pública con visión pedagógica y el espacio público como plataforma cultural que permite la coexistencia de la sociedad, en su diversidad de ideologías. Así, se entiende el museo como medio social, interactivo, ente pedagógico y experimento en el espacio-ciudad. Debido a la propuesta de museo urbano, el espacio público se analiza, a partir del reconocimiento y muestra de contenido urbano, artístico, histórico y patrimonial que pretende generar diálogo, crítica y contacto entre la gente.

\section{Experiencia: Valores del contenido}

El museo expone elementos urbanos vinculados en el trayecto, ya sean existentes en el espacio público delimitado o propuestos como parte de muestras temporales. Es en este sentido, que se entiende arquitectura y espacio público como lienzos urbanos, anuentes a ser experimentados e interpretados.

\section{Contenido Urbano}

-Componentes arquitectónicos: De interés patrimonial e histórico, en este caso se pueden valorar complejos edilicios, detalles arquitectónicos así como hitos.

-Material artístico-visual: Pretende exponer murales (en distintas técnicas), graffiti, intervenciones visuales, tanto existentes como propuestos. Esto es parte del esfuerzo por mostrar la imagen visual de la zona de estudio; que contempla distintos mensajes políticos, culturales e ideológicos provenientes de los habitantes.

-Expresiones individuales y colectivas: Acción y participación de distintos grupos de expositores (artistas, escritores, actores, otros), enfocado en el planteamiento de material crítico y cultural. Este tipo de contenido tiene potencial para ser expuesto temporalmente en espacios previamente analizados $\mathrm{y}$ seleccionados del recorrido.

Puede incluir material gráfico, sonoro, movimiento o intervenciones espaciales. El material gráfico puede presentarse mediante reproducciones, proyección u otras vías que permitan mayor oportunidad y accesibilidad para ser mostrado en el espacio público.

\section{Extensión de los museos}

Museos aledaños pueden participar en una escala distinta, extendiendo la exposición de su material (debidamente seleccionado para incentivar los planteamientos de Museo Urbano) al espacio público, lo que implica mayor alcance social. Mediante material audiovisual, gráfico, tridimensionalidades, intervenciones espaciales, entre otros.

E1 Museo Urbano, requiere del entendimiento de su contexto físico, social y temporal, lo que implica una fuerte conexión con las distintas muestras culturales que en su trayecto $-\mathrm{y}$ alrededores- se manifiesten. Lo anterior conlleva a la delimitación urbano-espacial, que permite reconocerlo en un sector específico de la ciudad, así, es posible que sea entendido y planteado según funciones de impacto social y pedagógico.

A partir de lo anterior y según lo desarrollado en la investigación, la propuesta cuenta con autonomía entre sus funciones y cuerpos. De esta manera, espacios formales como museos y galerías, tienen potencial como soporte de actividades relacionadas con el mantenimiento del material; además funciones de almacenamiento, selección y preparación del contenido.

Las implicaciones ya mencionadas, generan impacto desde infraestructura urbana, protección climática, mobiliario urbano, hasta 
elementos de apoyo o exposición (que permiten reconocer o mostrar el contenido del museo urbano) permanentes y temporales.

El museo urbano tiene razón de ser, no solo en la extensión o similitud con el museo ya establecido, sino con el espacio público como plataforma interactiva; dotada de material cultural, arquitectónico, histórico, político e ideológico, con la capacidad de enseñar y aprender. (Imagen 2)

\section{Eje peatonal Avenida Central: Laboratorio de Análisis}

El eje peatonal de la Avenida Central en San José, fue tomado en cuenta como laboratorio de análisis, para determinar sus condiciones como cuerpo físico y simbólico inmerso en un ambiente urbano; con el fin de establecer qué tipo de condiciones presenta para la propuesta del planteamiento Museo Urbano en sitio.

La estrategia de análisis, estuvo constituida mediante el acercamiento y observación del sitio en dos escalas distintas, macro y mesomicro, con el fin de entender oportunidades y problemáticas que se manifestaban tanto a nivel de centro urbano como de contacto humano en la vida pública.

Inicialmente el apartado macro, permitió entender la relación físico-urbana del eje peatonal con diferentes usos, hitos, nodos, sendas peatonales, espacios culturales y con respecto a las delimitaciones espaciales propuestas por el Plan de Acción para Centro Histórico de San José, como parte de los antecedentes de investigación. De esta manera se analizó la conexión y exposición cultural en el sitio, además del vínculo que mantiene con otras funciones.

En el bloque de análisis meso-micro, la estrategia se planteó a partir de una serie de cuestionamientos, que direccionaron los métodos de recopilación de información y análisis.

- ¿Cómo funciona la Avenida Central, quién comunica y qué se comunica? (desde la perspectiva cultural)

- ¿Cómo comunicar una ruta de connotación y acontecimientos culturales en el sitio?

- ¿Qué material urbano debe ser valorado y visibilizado en el sitio?

Según lo anterior, se llevó a cabo la búsqueda de respuestas o acercamientos, mediante el estudio de actividades e interacción en el sitio,

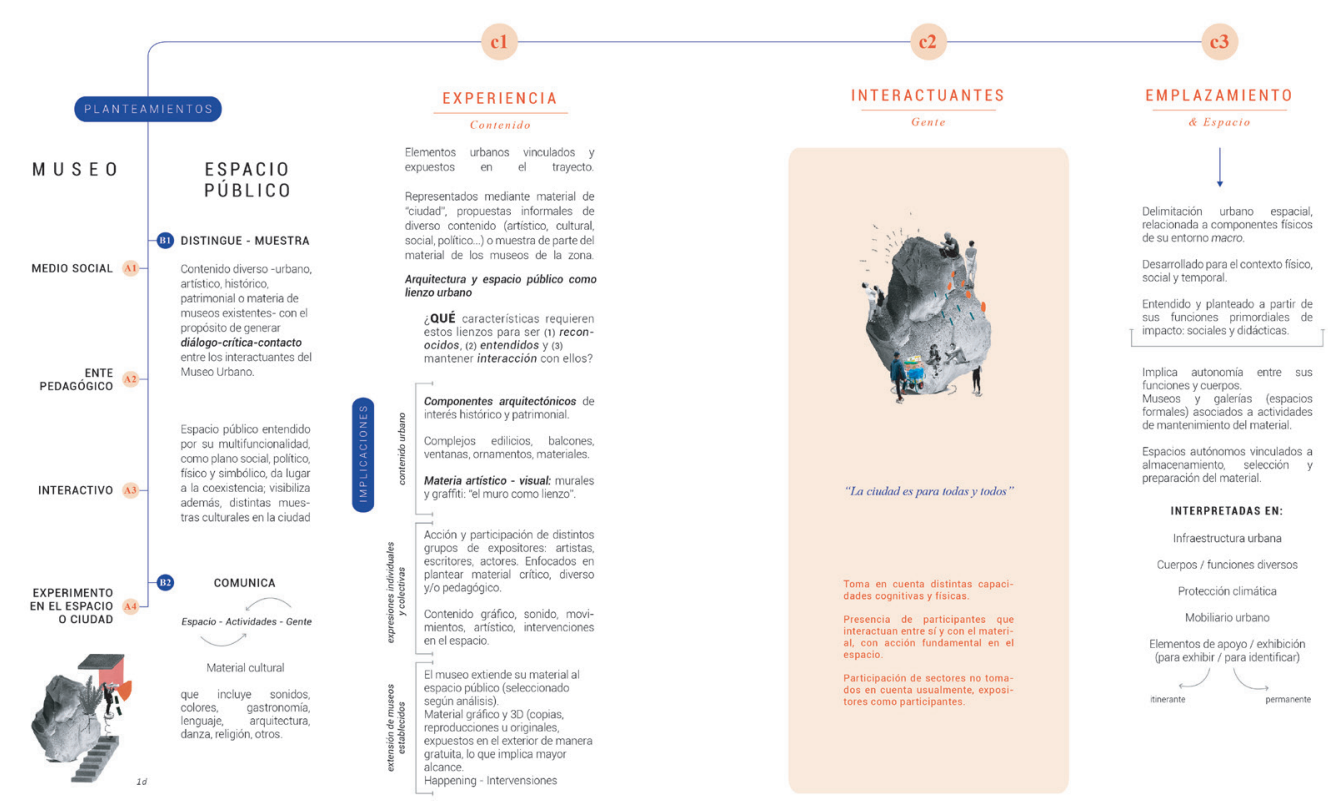

Fig. 2. (Imagen 2) Esquema de lógica: Museo Urbano (Rodríguez, Sofía. 2018) 
flujos peatonales, vehiculares, caracterización de las condiciones físico-urbanas, además de una reflexión perceptual sobre espíritu del lugar y la elaboración de imaginarios.

Cada categoría previamente mencionada, se estableció con el propósito de identificar la situación actual y modo de vibrar de la avenida, y su desarrollo se ejecutó mediante distintas herramientas para recopilar datos; tales como conteos de flujos peatonales, mapeos, registro documental y fotográfico.

A modo de síntesis, se realizó un cruce de información que permitió diagnosticar la Avenida Central en términos de conexión e interacción con sus manifestaciones culturales; con el fin de delimitar la zona de estudio a nivel micro para efectos del capítulo de planteamiento en sitio.

Lo que permitió establecer categorías de intervención para el eventual planteamiento en sitio, definidas en ejes:

-Actividades tomando en cuenta estancias, manifestaciones / expresiones en el espacio público y actividad comercial formal e informal -Vida Peatonal y transporte público -Condiciones físico-urbanas del medio

\section{Estrategias en sitio, a partir del planteamiento}

Como aproximación a los ejes previamente expuestos, se plantearon estrategias de acción para implementar Museo Urbano en un espacio público establecido, en este caso la Avenida Central en San José.

Se desarrolló un plan estratégico con la programación de intervenciones por cuadrante, en el tramo delimitado entre calles 1 y 17 del eje peatonal en San José, con el fin de evidenciar y proponer situaciones/condiciones que potencien la vida urbana. Las intervenciones fueron planteadas a partir de actividades, valorando dinámicas de interacción del eje y zonas aledañas, que además fueron expuestas en una tabla con sugerencias de tratamiento y observaciones de diseño.

Seguidamente, se valoró el planteamiento de conexiones entre espacios de encuentro y museos, como parte del concepto Paseo Cultural. Esto contempló tácticas que vinculan y traslapan intenciones de entes públicos, privados y organizaciones -que pretenden generar activación urbana-; a través de la toma de pequeñas acciones que intervienen escenas cotidianas en la ciudad.

\section{Aportes de investigación: Museo Urbano, óptica de lectura al espacio público}

Finalmente se desarrolló una serie de conclusiones que se identificaron a partir de la construcción conceptual y su posible impacto en el medio urbano.

Visión: Museo Urbano parte de la óptica con que se valora y entiende al museo, más allá de este, los espacios de exposición - artísticos o culturales -; como sitios "dispuestos" e incluso atentos a colaborar en la muestra de su contenido. Asimismo, le atribuye al espacio público estrategias mediante las cuales identifica, señala y comparte situaciones o elementos urbanos; que narran parte de la realidad social, cultural, política o histórica de la ciudad.

Lo pedagógico como experiencia: MUR se posiciona como vehículo a la comunicación, para exponer y narrar distintos tipos de contenido, enfocando su visión en compartir material de acceso para todas y todos en el espacio público, por lo tanto se aleja del esquema de objeto comerciable. Otorga a la burbuja pública la intención de manejar el material de exposición a partir de experiencias que evoquen los sentidos y la interacción social.

Experimento en la ciudad/espacio: El planteamiento Museo Urbano parte de la lógica de articulación entre diferentes cuerpos y funciones (públicas y privadas), así se entiende el espacio público como red de conexión evolutiva, vinculada a su contexto físico, simbólico, histórico, entre otros. Lo que pretende incentivar la apropiación del mismo mediante la experimentación en el espacio urbano; a partir de respuestas versátiles, flexibles y atemporales.

\section{Interacción: El museo es participación, no requiere audiencia.}

Da lugar al intercambio de acciones, las que la ciudad ofrece de manera clara y las que la 
gente desarrolla de acuerdo a sus necesidades; razón por la cual visibiliza actividades como ventas ambulantes, presentaciones artísticas, happenings, entre otras.

Toma en cuenta la existencia de diferentes capacidades cognitivas, de esta manera insta a la presencia de variados canales de exposición (visual, auditivo, sonoro y táctil), con el fin de permitir el acercamiento entre lo que se expone y quienes habitan el espacio público.

Estrategias de valoración y estudio: A partir de la concepción de MUR, se establece un cuerpo teórico que además de ser definido en términos de alcance conceptual, permite identificar insumos para estudiar el espacio público en que se pretenda aplicar. Las estrategias mencionadas anteriormente, pueden ser tomadas en cuenta como esquema metodológico interpretable para analizar la "vida pública" y algunas condiciones físicourbanas del medio.

Vida e identidad urbana: El carácter y esencia del Museo Urbano, parten de su conformación como estructura de distintas dimensiones, caracterizada por una identidad propia, vinculada a las dinámicas sociales que esta posea, según el contexto en que se encuentre inmersa. De modo que, la aplicación del planteamiento en el análisis de otros centros urbanos, encontrará cuestionamientos y soluciones distintas; que se acoplen a las manifestaciones culturales, económicas, sociales, entre otras, de ese entorno.

\section{Agradecimientos}

Agradezco a las personas que me guiaron en este proceso, Dra. arquitecta Zuhra Sasa, quién direccionó la investigación y sobre todo, me motivó a creer en el poder de las ideas. M.Sc. arquitecto Mario Villalta y Lic. arquitecto Rafael Venegas que desde sus experiencias y especialidades me guiaron, aportando a lo largo del proceso. También es necesario mencionar al urbanista M.Sc Daniel Morgan, agradezco su colaboración al brindarme material como habitante e investigador de la Avenida Central, pero esencialmente como guía en mi formación académica. A mi madre Silvia, por su apoyo inquebrantable a lo largo de la vida.

\section{Referencias}

Borja, Jordi y Muxí, Zaida. 2000. El espacio público, ciudad y ciudadanía. Barcelona. Disponible en: https://www.researchgate. net/publication/44358990_El_espacio publico_ciudad_y_ciudadania_Jordi_ Borja_y_Zaida_Muxi

Brandão, Pedro. 2011. La imagen de la ciudad: estrategias de identidad y comunicación. Barcelona: Universitat de Barcelona.

Delgado, Manuel. 2011. El espacio público como ideología. Madrid: Catarata.

Gehl, Jan. 2013. La humanización del espacio urbano: La vida social entre los edificios. Copenhague: Reverté.

León, Aurora. 2010. El museo: teoría, praxis y utopía. Madrid: Ediciones Cátedra.

Montaner, Josep M. 2003. Museos para el Siglo XXI. Barcelona: Editorial Gustavo Gili. 